\title{
Determinantes da eficiência de escala no setor brasileiro de operadores logísticos
}

\author{
Peter Fernandes Wanke, ${ }^{a, *}$, Camila Rodrigues Affonso ${ }^{\mathrm{b}}$ \\ a,*peter@coppead.ufri.br, COPPEAD/UFRJ, Brasil \\ bcamila.affonso@ilos.com.br, ILOS/UFRJ, Brasil
}

\begin{abstract}
Resumo
0 artigo foca o setor brasileiro de operadores logísticos (OLs) que, frente a um cenário altamente competitivo, oferece a seus clientes uma gama de serviços e de tecnologias de informação em busca de níveis maiores de eficiência. Seu objetivo principal é determinar as variáveis que apresentam impacto significativo na eficiência de escala dos OLs, por meio de modelagem de DEA (Data Envelopment Analysis - análise envoltória de dados) em dois estágios. Dados (2001-2008) da pesquisa anual feita pela Revista Tecnologística foram utilizados em um modelo de painel incompleto. Os inputs e outputs necessários para essa análise foram identificados, assim como as variáveis contextuais que podem impactar na eficiência de escala dos OLs. Os resultados corroboram evidências na literatura sobre o papel dos processos de coordenação no desempenho logístico.
\end{abstract}

Palavras-chave

DEA. Operadores logísticos. Eficiência de escala. Painel de dados.

\section{Introdução}

No Brasil, a indústria de operadores logísticos (OLs) começou a ganhar força a partir da estabilização econômica decorrente da implantação do Plano Real (FLEURY; RIBEIRO, 2003). Atualmente, dois terços dos gastos de cunho logístico das empresas brasileiras são destinados ao pagamento de prestadores de serviços logísticos, dado que evidencia a importância da terceirização para o país (CEL, 2009). Nesse sentido, os OLs devem buscar continuamente formas para se manter competitivos, sendo técnicas de avaliação de eficiência fundamentais nessa busca.

Especificamente, a técnica de análise envoltória de dados (Data Envelopment Analysis, DEA), desenvolvida há mais de 30 anos (COOK; SEIFORD, 2009), é considerada uma poderosa ferramenta para medir a eficiência, sobretudo por ser capaz de processar simultaneamente múltiplos inputs e outputs e proporcionar aos gestores um meio de apoio à tomada de decisão. Em conjunto com técnicas multivariadas de análise de dados, a DEA permite medir o impacto de variáveis contextuais nos níveis de eficiência (COOPER; SEIFORD; TONE, 2007).
Este artigo foca o setor brasileiro de OLs, com o objetivo de identificar os principais determinantes de sua eficiência de escala. Para isso, foi realizada uma revisão de literatura, não só no sentido de caracterizar o setor, mas também de justificar a modelagem adotada em dois estágios. Mais precisamente, ao cálculo da eficiência pela técnica de DEA, seguiu-se a regressão Tobit com dados em painel incompleto, permitindo medir o impacto de variáveis contextuais na eficiência de escala do setor. Os resultados corroboram o impacto positivo de processos de coordenação, baseados no uso de tecnologias de informação e no controle de estoques, no desempenho logístico.

0 restante do artigo está dividido em cinco seções. A seção 2 discute o papel dos OLs nas cadeias de suprimento, os principais serviços prestados e as tecnologias de informação passiveis de serem adotadas. Também são apresentados os poucos estudos anteriores que aplicaram DEA ao setor de OLs em outros países. Na seção 3, a modelagem de DEA em dois estágios é apresentada mais detalhadamente, justificando-se, então, a 
escolha da eficiência de escala como meio de se avaliar o impacto dos processos de coordenação no desempenho logístico. Na seção 4 , os dados são analisados e os resultados, discutidos. Finalizando o artigo, a seção 5 apresenta as conclusões.

\section{Revisão de literatura}

Como reflexo da popularização da terceirização logística no meio empresarial e do subsequente crescimento dos serviços contratados com prestadores de serviço, um grande volume de artigos e pesquisas foi conduzido nos últimos anos, com o objetivo de entendimento maior dos diferentes aspectos relacionados aos OLs. Esses aspectos incluem, por exemplo, a sua conceituação, as motivações para sua contratação e o escopo das atividades prestadas por eles (ZHOU et al., 2008).

Em linhas gerais, os OLs são:

[...] fornecedores de serviços logísticos integrados, capazes de atender a todas ou quase todas as necessidades logísticas de seus clientes, de forma personalizada" (FLEURY, 2000).

Dentre alguns dos motivadores para a onda de terceirização dos serviços logísticos e para a contratação de OLs, destacam-se a redução de custos, a melhoria nos níveis de serviço, o aumento da flexibilidade operacional e o foco nas competências-chave do negócio (WILDING; JURIADO, 2004). Baseando-se na diversidade de razões para terceirizar partes da operação logística, é de se esperar o surgimento de OLs capacitados a desempenhar múltiplas tarefas com diferentes níveis de especialização (FIGUEIREDO; MORA, 2009).

Em survey conduzido no Brasil com grandes empresas manufatureiras contratantes dos serviços de OLs, Wanke, Arkader e Hijar (2007) identificaram forte associação entre a estrutura do processo produtivo dessas empresas e o tipo de serviços e do arcabouço tecnológico oferecido pelos OLs. Mais especificamente, foi evidenciado que empresas dos setores automotivo, eletroeletrônico e aeroespacial tendem a contratar OLs integrados, ou seja, que gerenciam transporte, armazenagem e estoques de forma simultânea e com uso intensivo de tecnologias de informação. Por outro lado, empresas dos setores de alimentos, de bebidas e de combustíveis, por exemplo, tendem a contratar OLs de menor base tecnológica, fundamentalmente concentrados na prestação de serviços de transporte.

A implantação de complexas tecnologias de informação é cada vez mais comum em OLs que coordenam uma ampla gama de atividades para seus clientes. Nesses casos, a transmissão da "[...] informação correta, para a pessoa correta, no momento correto e de forma que possa ser utilizada em tempo real" (YOUNGBERG; OLSEN; HAUSER, 2009). é um dos principais desafios na prestação do serviço logístico. Em particular, os sistemas Enterprise Resource Planning - ERP padronizam e integram as informações de demanda, tornando-as mais confiáveis para o planejamento dos recursos de transporte e armazenagem do OL relativamente aos estoques do cliente, possibilitando, assim, a melhoria de seu desempenho operacional (CHOU; CHANG, 2008). Os OLs também são reconhecidos pelo mercado quando detêm o domínio de tecnologias de informação (Tls) mais sofisticadas e disponibilizam diversas informações via internet, como, por exemplo, a consulta do status da carga em trânsito (LIEB, R.C.; LIEB, K.J., 2008). Em suma, os clientes consideram o respaldo tecnológico como um item básico a ser fornecido pelos OLs (LIEB, 2005).

0 reconhecimento do impacto dos processos de coordenação da cadeia de suprimentos sobre o transporte e a armazenagem, atividades-chave desempenhadas pelos OLs, não é novo (NG; FERRIN; PEARSON, 1997). A novidade é o surgimento de aplicações de $\mathrm{Tl}$ que mudaram o modo de operação dessas atividades e alavancaram o desempenho da cadeia de suprimentos (MASON et al., 2003; STEFANSSON; LUMSDEN, 2009). Sistemas de gerenciamento de transporte e de armazenagem, por exemplo, são tecnologias-chave usadas para gerir o fluxo físico de mercadorias ao longo da cadeia de abastecimento. Sistemas integrados, que abrangem sistemas de gestão de transporte, sistemas de gerenciamento de armazenagem e visibilidade global de estoque através da internet, podem potencialmente conduzir à redução de custos e à melhoria no serviço ao cliente através de melhor adequação entre recursos e demandas, com a diminuição de tempos de ciclo de envio e recebimento, o aumento da precisão no embarque e a diminuição da variabilidade nos tempos de resposta (MASON et al., 2003).

Os processos de certificação, como aqueles desenvolvidos pela International Organization for Standardizations - 1S0, são outro quesito valorizado na indústria de OLs. A certificação permite elevar os níveis de serviço prestados, por meio da estruturação e implantação de procedimentos padronizados. Foi empiricamente comprovado que a 150 9000, por exemplo, melhora o desempenho das operações logísticas, apresentando resultados positivos logo após sua adoção. Esse melhor desempenho se traduz em um intervalo de tempo mais curto para a circulação de produtos e dinheiro entre fornecedores, clientes e prestadores de serviço (LO; YEUNG; CHENG, 2009).

De acordo com Zhou et al. (2008), apesar dos numerosos estudos sobre o setor de OLs conduzidos 
até então, apenas dois tiveram por objetivo avaliar o desempenho dessa indústria por meio da técnica de DEA, o que claramente sugere uma lacuna a ser preenchida. Deve ser ressaltado, entretanto, que essa técnica já foi empregada de maneira satisfatória em outros segmentos que lidam diretamente com logística, como, por exemplo, em empresas do setor aéreo (SCHEFCZYK, 1993), aeroportos (PACHECO; FERNANDES, 2003), transportadores rodoviários de passageiros (ODECK; ALKADI, 2001), terminais de contêineres (WANG; SONG; CULLINANE, 2002; TURNER; WINDLE; DRESSNER, 2004; MIN; PARK, 2005), portos de maneira geral (PANAYIDES et al., 2009) e grandes redes distribuidoras de petróleo (ROSS; DROGE, 2004).

Conforme comentado, os estudos que relatam a aplicação de DEA, especificamente no setor de OLs, são escassos e recentes. Min e Joo (2006), por exemplo, aplicaram a técnica a um grupo de seis OLs, selecionados dentre os maiores dos Estados Unidos. Os autores construíram um benchmark como forma de identificar os OLs que desenvolvem boas práticas e permitir que outros possam espelhar-se nelas. Segundo os autores, a técnica de DEA auxilia a guiar os investimentos financeiros, bem como a medir seus impactos no desempenho da empresa. Os resultados indicaram que OLs americanos, que figuraram na lista dos 25 maiores em 2000, não se revelaram eficientes em qualquer momento do período investigado (1999-2002). Constatou-se também que a queda no crescimento da indústria manufatureira americana, em 2001, ocasionou um declínio no desempenho operacional dos OLs estudados.

Hamdan e Rogers (2007) aplicaram a técnica de DEA às operações de armazenamento dos OLs. Foram estudados 19 armazéns de um OL americano. 0 estudo reflete a importância dos processos de armazenagem para o setor. Para efeitos de modelagem, foram selecionados insumos, que mostrassem trabalho, espaço, tecnologia e equipamentos, e resultados, que refletissem quantidade produzida, atendimento de pedidos e utilização do espaço. As análises foram validadas pelo OL, na medida em que quatro armazéns, dos seis classificados como eficientes, figuravam entre aqueles de mais alto desempenho da empresa.

Por sua vez, Zhou et al. (2008) aplicaram a técnica DEA ao setor de OLs da China. Sua intenção não era somente estabelecer um benchmark para o setor, mas também identificar fatores que pudessem impactar no desempenho dos OLs. Para isso, após serem medidos os níveis de desempenho operacional do grupo investigado, conduziu-se uma análise de regressão dos escores obtidos contra quatro possiveis fatores impactantes. Dentre as principais conclusões, destaca-se o fato de que o tamanho de uma companhia pode não impactar positivamente na eficiência do OL, conforme seria o esperado. Descobriu-se ainda que a receita acumulada de vendas permite melhor utilização dos recursos do OL e que investimentos em treinamento da equipe de funcionários, além de reter pessoal capacitado, influenciam positivamente no desempenho do OL.

Em linhas gerais, o grande desafio que se impõe aos estudos que aplicam DEA em empresas de logística é a identificação de fatores ambientais ou variáveis contextuais que impactam significativamente na eficiência (ZHOU et al., 2008). Neste artigo, o setor brasileiro de OLsé analisado no período de 2001-2008, por meio de um modelo de DEA em dois estágios, o qual envolve primeiramente a determinação dos escores de eficiência, seguida da análise de dados em painel incompleto por meio de regressão Tobit. A modelagem é apresentada a seguir.

\section{Modelagem de DEA em dois estágios}

DEA é um método não paramétrico primeiramente introduzido por Charnes, Cooper e Rhodes (1978). Apesar dos seus mais de 30 anos, essa técnica continua recebendo grande atenção do meio acadêmico (COOK; SEIFORD, 2009). É baseada em programação linear e usada para calcular a eficiência relativa de um grupo de unidades de negócio (Decision Making Units - DMUs), por meio da utilização de várias medidas de inputs e outputs. Dado um conjunto de DMUs, inputs e outputs, a DEA determina para cada DMU uma medida de eficiência obtida como uma razão entre outputs ponderados e inputs ponderados. Existem diversas variações da técnica, diferindo, por exemplo, no que diz respeito a ganhos de escala e ao modo como é calculada a distância de DMUs ineficientes até a fronteira (ZHU, 2003).

Suponhamos $s=1 . . S$ unidades de produção, com $x_{\mathrm{s}}^{\mathrm{T}}=\left(x_{\mathrm{s} 1}, \ldots, x_{\mathrm{sm}}\right)$ inputs e $y_{\mathrm{s}}^{\mathrm{T}}=\left(y_{\mathrm{s} 1}, \ldots ., y_{\mathrm{sn}}\right)$ outputs. Os vetores-coluna $x_{\mathrm{s}}$ e $y_{\mathrm{s}}$ formam a $s$-ésima coluna das matrizes $X$ e $Y$. Suponhamos, além disso, que $\lambda^{\mathrm{T}}=\left(\lambda_{1}, . ., \lambda_{\mathrm{s}}\right)$ é um vetor não negativo e que $\mathrm{e}^{\mathrm{T}}=(1, . ., 1) \in R^{\mathrm{s}}$ é um vetor de valores unitários. Os modelos DEA-CCR (CHARNES; COOPER; RHODES, 1978) e DEA-BCC (BANKER; CHARNES; COOPER, 1984) são mostrados nas Equações (1) e (2):

$$
\begin{aligned}
& \text { DEA-CCR } \\
& \text { Orientado a Input } \\
& \text { min }_{\theta, \lambda} \theta \\
& \text { s.t. } \theta x_{s}-X \lambda \geq 0 \\
& Y \lambda \geq y_{s} \\
& \lambda \geq 0
\end{aligned}
$$


$D E A-B C C$

Orientado a Input

$\min _{\theta, \lambda} \theta$

s.t. $\theta x_{s}-X \lambda \geq 0$

$Y \lambda \geq y_{s}$

$e \lambda=1$

A maior vantagem da DEA em relação aos métodos paramétricos é que os pesos relativos das variáveis não precisam ser conhecidos a priori. Sua fronteira eficiente está sobre o limite de um de um poli-topo convexo criado no espaço de inputs e outputs, no qual cada vértice é uma DMU eficiente (DULÁ; HELGASON, 1996). Além de proporcionar medidas de eficiência, a DEA também oferece outras informações relevantes para as DMUs ineficientes. A DEA identifica a faceta eficiente sendo usada para comparação, a combinação de inputs que estão sendo usados de forma ineficiente, e o desvio de outputs específicos a partir do nível eficiente. Deve ser notado que DMUs eficientes tendem a não apresentar qualquer folga, sendo essa informação relatada unicamente para DMUs ineficientes (GREEN; DOYLE; COOK, 1996; LIN; TSENG, 2007).

A ineficiência de escala se deve ao aumento ou à redução dos retornos de escala, o que pode ser determinado inspecionando a soma dos pesos de acordo com as especificações do modelo CCR. Se essa soma é igual a 1, a lei de retornos constantes de escala prevalece, mas, se a soma for inferior ou superior a 1, respectivamente, retornos crescentes de escala e retornos decrescentes de escala prevalecem, considerando-se um modelo orientado a inputs. Ainda de acordo com Cooper, Seiford e Tone (2007), para identificar quanto da ineficiência de uma DMU é causado pela realização de operações ineficientes ou por seu tamanho de escala, define-se a eficiência de escala por meio da seguinte razão (Equação 3):

$$
E E=\frac{\theta_{C C R}}{\theta_{B C C}}
$$

É importante destacar que o valor máximo de $E E$ é 1, indicando que a DMU está operando no tamanho de escala mais produtivo.

Neste estudo, nosso interesse pela eficiência de escala passa por determinar não apenas se há algum OL operando em seu nível ótimo, ou próximo dele, dado o conjunto de inputs empregado e o nível de outputs gerado, mas também por determinar as condições objetivas para que isso aconteça de modo análogo ao estudo de Ross e Droge (2004). Em outras palavras, a eficiência de escala pode ser empregada para determinar o quão próximo cada um dos OLs da amostra está de seu correspondente tamanho de escala mais produtivo, e quanto isso é reflexo de seus processos de coordenação na cadeia de suprimentos: gestão dos fluxos de informações, controle de estoques e dimensionamento de recursos (WANKE, 2003). Em sistemas de distribuição de larga escala (situação que é característica dos OLs), diferentes processos de coordenação frequentemente levam a diferentes padrões de alocação de recursos por entre atividades, podendo tornar mais flexível a adequação da escala à operação (ROSS; DROGE, 2004). Nesse caso, os resultados da eficiência de escala podem indicar oportunidades para downsizing (retornos decrescentes de escala) ou consolidação de operações (retornos crescentes de escala). Por exemplo, dependendo dos usos alternativos das tecnologias de informação (Tls) e dos mecanismos para controlar e movimentar os estoques por parte dos OLs, pode haver situações em que o armazém experimenta retornos decrescentes (crescentes) de escala por ser muito grande (pequeno) comparativamente aos níveis de estoque, de movimentação de carga e de demanda que por lá são alocados (ROSS; DROGE, 2004). A ideia básica é, portanto, verificar o papel desses processos de coordenação na determinação da eficiência de escala do OL, avaliando se, de fato, eles proporcionam uma alocação mais racional dos recursos (inputs) à demanda (outputs) e, consequentemente, uma operação próxima do tamanho de escala mais produtivo, tendo como pano de fundo a disponibilização de informação em tempo real.

As abordagens para tratamento estatístico das variações nos escores gerados pela técnica de DEA têm evoluído ao longo dos anos, vide, por exemplo, os artigos de Banker (1993) e Simar e Wilson (2007). Como retrato dessa evolução, Cooper, Seiford e Tone (2007) apontam um número crescente de estudos que combinam entre si os resultados gerados pela DEA, numa primeira etapa, com análises multivariadas de dados, como análise de regressão e análise estocástica da fronteira (Stochastic Frontier Analysis - SFA), numa segunda etapa. Segundo Fried et al. (2002), essas abordagens de DEA em dois estágios (two-stage DEA approaches) são fruto do reconhecimento, por parte dos pesquisadores, de que fatores ambientais ou variáveis contextuais podem influenciar de forma significativa nos escores de eficiência. Por exemplo, segundo esses autores, a competência (ou incompetência) gerencial não é suficiente para explicar individualmente variações nos níveis de eficiência, dado que fatores ambientais, variáveis contextuais ou, até mesmo, ruídos estatísticos poderiam exercer alguma influência sobre o desempenho medido. 0 controle adequado desses impactos poderia indicar possíveis caminhos para uma DMU se tornar mais eficiente (SOUZA et al., 2007). 
Neste artigo, a análise multivariada do segundo estágio fará uso de regressão Tobit aplicada a um painel incompleto de dados referentes à indústria brasileira de OLs, no período de 2001-2008. Esses dados são apresentados mais detalhadamente na seção 4. De acordo com Turner, Windle e Dressner (2004), como a variável dependente (eficiência de escala) é contínua, mas truncada em 1, a abordagem por mínimos quadrados ordinários (Ordinary Least Squares - OLS) não é apropriada, já que pode produzir estimadores inconsistentes. Em linhas gerais, o caso base da regressão Tobit é similar à regressão por OLS, mas assume uma distribuição normal truncada no lugar da distribuição normal e emprega métodos de estimação de máxima verossimilhança (GREENE, 2007). Banker (1993), entretanto, também abre possibilidade para o uso de outras distribuições adequadas para a regressão Tobit, como a exponencial e a meia-normal.

Souza e Staub (2007) estenderam os resultados de Banker (1993), mostrando que os problemas de inferência na abordagem de DEA em dois estágios, apontados por Simar e Wilson (2007), não são gerais. De fato, procedimentos baseados em DEA com regressão Tobit no segundo estágio apresentam desempenho equivalente ao melhor dos métodos paramétricos na estimação do impacto de variáveis contextuais na produtividade (BANKER; NATARAJAN, 2008). Por fim, deve-se destacar que o uso de testes não paramétricos, como aqueles apresentados em Banker e Natarajan (2004) e Gomes et al. (2009), constitui alternativa tão comum quanto o uso de regressão Tobit em situações similares.

Modelos de dados em painel permitem o exame de efeitos fixos ou aleatórios de uma determinada empresa ou dos períodos de tempo nos escores de eficiência (PARK, 2005). No caso dos modelos de efeitos aleatórios - de acordo com Greene (2007) os mais frequentemente utilizados -, as premissas básicas são: o efeito aleatório $u_{\mathrm{i}}$ é o mesmo para todos os períodos e este não deve ser correlacionado com os demais regressores; os coeficientes angulares são os mesmos para todos os grupos e períodos; e $\varepsilon_{\mathrm{it}}$, o componente estocástico do modelo, não é correlacionado entre períodos. A forma funcional do modelo de efeitos aleatórios é dada pela Equação 4:

$y_{i t}=X_{i t} \beta+u_{i}+\varepsilon_{i t}$

onde:

- $i$ denota o grupo ou indivíduo;

- $t$ denota o período de tempo;

- $y_{\text {it }}$ denota a variável dependente; e

- $X_{\text {it }}$ denota o vetor de variáveis independentes.

\section{Análise de dados e discussão de resultados}

Tomando como ponto de partida a discussão precedente, este estudo pretende determinar os principais fatores que impactam na eficiência de escala da indústria de OLs no Brasil. Os dados utilizados foram coletados de edições especiais (2001-2008) da Revista Tecnologística, dedicadas a esse setor e publicadas nos meses de junho ou julho de cada ano. 0 fato de se trabalhar com dados secundários, e não com dados primários, traz algumas limitações ao trabalho, principalmente no que diz respeito ao conjunto de inputs e outputs para análise, os quais não necessariamente abrangem todos os aspectos relevantes para a determinação da fronteira de eficiência. Além disso, deve-se destacar que as bases de dados originais foram depuradas, descartando-se as variáveis independentes que não foram coletadas para todos os indivíduos listados no painel, de forma a deixar as informações homogêneas o suficiente para a execução dos modelos.

No primeiro estágio, os modelos de DEA, CCR e BBC foram executados oito vezes no software Frontier Analyst 4.0.10, ou seja, uma vez para cada um dos anos do período compreendido entre $2001 \mathrm{e}$ 2008. Mais especificamente, o painel incompleto de dados sobre a indústria brasileira de OLs contém 85 indivíduos, totalizando 164 observações distribuídas ao longo desses oito anos (Quadro 1).

Para construção dos modelos de DEA, foram selecionados inicialmente quatro inputs e dois outputs comuns a todos os Ols integrantes da pesquisa. Seguindo o exemplo de estudos anteriores (ZHOU et al., 2008), foram escolhidas unidades de medida que traduzissem recursos críticos não só financeiramente, mas também para consecução dos serviços. Com relação aos inputs, o número total de funcionários do OL, envolvidos tanto em atividades estratégicas quanto operacionais, é a medida empregada para traduzir a utilização da força de trabalho.

Além dessa, é necessária a seleção de medidas que traduzam como os OLs lidam com a armazenagem, já que ela é a atividade terceirizada que mais cresceu no período de 2001-2008 (MARINO, 2008). De acordo com a autora, a oferta de serviços de armazenagem é maior que a de transportes no setor de OLs. Nesse sentido, a área total de armazenagem e o total de armazéns próprios foram selecionados como inputs para o modelo. É importante considerar também as situações onde o OL opera o armazém, mas todos os ativos pertencem ao cliente (MARINO, 2008). Nesse caso, aquele armazém, que funciona como recurso 
Quadro 1. Painel incompleto de dados - Indústria brasileira de OLs.

\begin{tabular}{|c|c|c|c|c|c|c|c|c|c|c|c|c|c|c|c|c|c|}
\hline Empresa & 2001 & 2002 & 2003 & 2004 & 2005 & 2006 & 2007 & 2008 & Empresa & 2001 & 2002 & 2003 & 2004 & 2005 & 2006 & 2007 & 2008 \\
\hline $4 \mathrm{PL}$ & & & & & & & $x$ & & GAT & & & $x$ & & & & $x$ & \\
\hline Abrange & $x$ & & & & & & & & GTech & $x$ & & $x$ & $x$ & & & & \\
\hline AGM & & & & $x$ & $x$ & & $x$ & & Golden & & & & & & & & $x$ \\
\hline AGR & & & & & & & $X$ & $x$ & GPT & & & $x$ & & & & & \\
\hline Águia & $x$ & & & & & & & & Grandeabc & & & & & & & $x$ & \\
\hline AGV & $x$ & $x$ & & & & & $x$ & $x$ & Granvale & & & & & & & & $x$ \\
\hline Argimpel & $x$ & & & & & & & & Grecco & & & & $x$ & & & & \\
\hline Armavale & & $x$ & & & & & & & Intermar & & & & $x$ & $x$ & & $x$ & \\
\hline Atlas & & & & & & & $x$ & $x$ & Intermod & $x$ & & & & & & & \\
\hline Beta & & & & $x$ & & & & & 1tamarlog & & & $x$ & $x$ & $x$ & & $x$ & \\
\hline Binotto & & & & & & $x$ & $x$ & $x$ & KT\&T & & & $x$ & $x$ & & & $x$ & $x$ \\
\hline Brasiliense & & & $x$ & $x$ & & & $x$ & $x$ & Kuehnenagel & & & $x$ & $x$ & & & & $x$ \\
\hline Bravo & $x$ & $x$ & $x$ & $x$ & & $x$ & $x$ & & Lamounier & & & $x$ & & & & & \\
\hline Brasex & $x$ & & & & & & & & $\mathrm{LG}$ & $x$ & & & & & & & \\
\hline Brazilian & & & & & & & & $x$ & Libra & & & & & & & & $x$ \\
\hline Brilhante & $x$ & & & & & & & & Logisplan & & & $x$ & & & & & \\
\hline Bueno & & & & & & & & $x$ & Loghis & $x$ & & & & & & & \\
\hline CBCE & & & & & & & $x$ & & M3 & $x$ & & & & & & & \\
\hline Celere & & & & & & & $x$ & & MCP & $x$ & & & $x$ & $x$ & & $x$ & $x$ \\
\hline Cesa & & & & & & & $x$ & & Mercúrio & & & & & & & & $x$ \\
\hline Columbia & $x$ & $x$ & & & $x$ & & & & Mirassol & & & $x$ & & & & & \\
\hline Comint & & & & $x$ & $x$ & & $x$ & & Norlog & $x$ & & & & & & & \\
\hline Conseil & $x$ & & $x$ & & & & & & NSF & $x$ & $x$ & $x$ & & $x$ & & $x$ & $x$ \\
\hline Continental & & & & $x$ & & & & & Panazzolo & & & $X$ & & & & & \\
\hline Coopercarga & $x$ & & & & $x$ & & & & Panzan & & & & & & $x$ & $x$ & $x$ \\
\hline Cragea & $x$ & & & $x$ & & $x$ & & $x$ & Petrolog & & & $x$ & & & & $x$ & \\
\hline $\mathrm{CS} 1$ & & & & & & & & $x$ & Prolog & & & & & & & $x$ & \\
\hline Custom & $x$ & & & & & & & & Pronto & & & & & & & & $x$ \\
\hline Danzas & $x$ & $x$ & & & & & & & Rap900 & & & & & & & $x$ & $x$ \\
\hline Deicimar & $x$ & & & & & & & & Rodoborges & $x$ & & $x$ & $x$ & $x$ & $x$ & & \\
\hline Delara & $x$ & $x$ & & & & & $x$ & & Ryder & & & & & & & & $x$ \\
\hline DHL & $x$ & & & & & & & & Standart & & & & & & & & $x$ \\
\hline Drago & $x$ & & & & & & & & Sulista & & & & & & & & $x$ \\
\hline Dry Port & & & & & & & & $x$ & Stocktech & & $x$ & & & & & & \\
\hline EBA & & & & & & & $x$ & & SYN & $x$ & & $x$ & & & & $x$ & \\
\hline Eichenberg & $x$ & & & $x$ & & & & & TAC & & & & & & & $x$ & $x$ \\
\hline Elba & & & $x$ & & & & & & TDS & $x$ & $x$ & $x$ & $x$ & $x$ & & & \\
\hline Enar & $x$ & $x$ & & & & & & & Tegma & $x$ & & & & & & & $x$ \\
\hline Estrada & $x$ & $x$ & $x$ & $x$ & & & $x$ & $x$ & TNT & $x$ & & $x$ & & & & & \\
\hline Eudmarco & & & & & $x$ & & & & Ultracargo & & & & & & & $X$ & $x$ \\
\hline Explimeira & & & & $x$ & $x$ & & & & UPS & & & & $x$ & & & & $x$ \\
\hline Flexil & & & $x$ & & & & & $x$ & Vale Log-ln & & & & & & & $x$ & \\
\hline Fluxo & & & $x$ & & & & & & & & & & & & & & \\
\hline
\end{tabular}

operacional do OL sem, no entanto, pertencer ao mesmo, é computado na medida do total de armazéns de clientes, constituindo o último input do modelo.

Pelo lado dos outputs, foram selecionadas inicialmente medidas que traduzissem aspectos financeiros e operacionais. Dessa forma, a receita bruta do OL retrata o produto da venda dos serviços prestados, sendo selecionada como output. 0 número total de clientes da empresa, de modo semelhante, reflete sua complexidade operacional, já que uma ampla carteira de clientes pode indicar não só boa imagem no mercado, mas também habilidade no gerenciamento de diferentes necessidades de serviço logístico.

Vários métodos têm sido propostos na literatura sugerindo a limitação do número de variáveis em relação ao número de DMUs (WAGNER; SHIMSHAK, 2007). Alguns estudos sugeriram julgamento por especialistas, a fim de indicar as variáveis mais relevantes para o modelo DEA (GOLANY; ROLL, 1989); análise de regressão, a fim de indicar as variáveis altamente correlacionadas como redundantes 
(LEWIN; MOREY; COOK, 1982); ou aplicação da DEA para modelos reduzidos, a fim de classificar o efeito de variáveis sobre os escores de eficiência (WAGNER; SHIMSHAK, 2007). Na Tabela 1, são apresentados os coeficientes de correlação entre os pares de inputs e o par de outputs. Como as correlações seriais são relativamente baixas, optamos por manter todos os inputs e outputs na análise.

$\mathrm{Na}$ Tabela 2, são apresentadas as estatísticas descritivas para os escores determinados pelos modelos CCR e BCC e para a eficiência de escala no período 2001-2008. Conforme esperado, os modelos CCR retornam escores de eficiência menores do que aqueles determinados por meio dos modelos BCC. Em outras palavras, os modelos CCR identificam menos OLs eficientes do que os modelos BCC para cada ano. Esse resultado não é surpreendente, pois o modelo CCR pressupõe uma tecnologia de produção com retornos constantes (lineares) de escala, enquanto o modelo BCC sugere retornos variáveis de escala, que são mais aderentes à realidade por refletirem a eficiência técnica de diferentes DMUs.

Tabela 1. Correlações entre inputs e outputs.

\begin{tabular}{|c|c|c|c|c|c|c|}
\hline & \multicolumn{4}{|c|}{ Inputs } & \multicolumn{2}{|c|}{ Outputs } \\
\hline & $\begin{array}{l}\text { Número de } \\
\text { funcionários }\end{array}$ & $\begin{array}{c}\text { Área de } \\
\text { armazenagem } \\
\text { total }\end{array}$ & $\begin{array}{c}\text { Total de } \\
\text { armazéns } \\
\text { próprios }\end{array}$ & $\begin{array}{l}\text { Total de } \\
\text { armazéns de } \\
\text { clientes }\end{array}$ & $\begin{array}{l}\text { Número de } \\
\text { clientes }\end{array}$ & Receita bruta \\
\hline Número de funcionários & 1,00 & & & & & \\
\hline Área de armazenagem total & 0,45 & 1,00 & & & & \\
\hline Total de armazéns próprios & 0,16 & 0,38 & 1,00 & & & \\
\hline Total de armazéns de clientes & 0,51 & 0,36 & 0,13 & 1,00 & & \\
\hline Número de clientes & & & & & 1,00 & \\
\hline Receita bruta & & & & & 0,03 & 1,00 \\
\hline
\end{tabular}

Tabela 2. Resumo das eficiências calculadas por ano.

\begin{tabular}{|c|c|c|c|c|c|c|c|c|c|c|}
\hline Escore & Ano & 2001 & 2002 & 2003 & 2004 & 2005 & 2006 & 2007 & 2008 & Todos \\
\hline \multirow{7}{*}{ CCR } & Média & 0,19 & 0,57 & 0,43 & 0,62 & 0,53 & 0,45 & 0,44 & 0,53 & 0,44 \\
\hline & Mínimo & 0,00 & 0,15 & 0,06 & 0,10 & 0,20 & 0,14 & 0,05 & 0,09 & 0,00 \\
\hline & Máximo & 1,00 & 1,00 & 1,00 & 1,00 & 1,00 & 1,00 & 1,00 & 1,00 & 1,00 \\
\hline & Desvio padrão & 0,28 & 0,33 & 0,34 & 0,31 & 0,30 & 0,33 & 0,36 & 0,33 & 0,35 \\
\hline & Coeficiente de variação & 1,47 & 0,59 & 0,80 & 0,50 & 0,57 & 0,74 & 0,82 & 0,62 & 0,78 \\
\hline & Qte. de DMUs eficientes & 3 & 3 & 4 & 5 & 2 & 1 & 6 & 7 & 31 \\
\hline & \% de DMUs eficientes & 9 & 27 & 17 & 25 & 17 & 20 & 20 & 23 & 19 \\
\hline \multirow{7}{*}{$\mathrm{BCC}$} & Média & 0,70 & 0,87 & 0,87 & 0,80 & 0,83 & 0,63 & 0,65 & 0,77 & 0,76 \\
\hline & Mínimo & 0,17 & 0,37 & 0,47 & 0,25 & 0,25 & 0,31 & 0,06 & 0,24 & 0,06 \\
\hline & Máximo & 1,00 & 1,00 & 1,00 & 1,00 & 1,00 & 1,00 & 1,00 & 1,00 & 1,00 \\
\hline & Desvio padrão & 0,29 & 0,21 & 0,19 & 0,28 & 0,31 & 0,25 & 0,34 & 0,27 & 0,33 \\
\hline & Coeficiente de variação & 0,42 & 0,24 & 0,22 & 0,35 & 0,37 & 0,39 & 0,52 & 0,35 & 0,44 \\
\hline & Qte. de DMUs eficientes & 11 & 10 & 9 & 12 & 11 & 4 & 13 & 11 & 81 \\
\hline & \% de DMUs eficientes & 33 & 91 & 39 & 60 & 92 & 80 & 43 & 37 & 49 \\
\hline \multirow{7}{*}{ EE } & Média & 0,24 & 0,65 & 0,48 & 0,78 & 0,65 & 0,71 & 0,63 & 0,70 & 0,57 \\
\hline & Mínimo & 0,01 & 0,21 & 0,06 & 0,27 & 0,24 & 0,26 & 0,17 & 0,09 & 0,01 \\
\hline & Máximo & 1,00 & 1,00 & 1,00 & 1,00 & 1,00 & 1,00 & 1,00 & 1,00 & 1,00 \\
\hline & Desvio padrão & 0,28 & 0,32 & 0,34 & 0,26 & 0,26 & 0,32 & 0,30 & 0,32 & 0,34 \\
\hline & Coeficiente de variação & 1,13 & 0,49 & 0,70 & 0,33 & 0,39 & 0,46 & 0,48 & 0,46 & 0,61 \\
\hline & Qte. de DMUs eficientes & 3 & 3 & 4 & 6 & 2 & 1 & 6 & 7 & 32 \\
\hline & \% de DMUs eficientes & 9 & 27 & 17 & 30 & 17 & 20 & 20 & 23 & 20 \\
\hline \multicolumn{2}{|c|}{ Total de DMUs } & 33 & 11 & 23 & 20 & 12 & 5 & 30 & 30 & 164 \\
\hline \multicolumn{2}{|c|}{ Qte. de DMUs - CRS } & 11 & 10 & 9 & 12 & 11 & 4 & 13 & 11 & 81 \\
\hline \multicolumn{2}{|c|}{ Qte. de DMUs VRS - Crescente } & - & 0 & 1 & 1 & 0 & 0 & 1 & 1 & 4 \\
\hline \multicolumn{2}{|c|}{ Qte. de DMUs VRS - Decrescente } & 22 & 1 & 13 & 7 & 1 & 1 & 16 & 18 & 79 \\
\hline
\end{tabular}

CRS = Retorno constante de escala; VRS = Retorno variável de escala. 
Além disso, percebe-se que muito poucos OLs operam no tamanho de escala mais produtivo.

Com o objetivo de identificar os determinantes da eficiência de escala dos OLs atuando em território nacional, foram pesquisados, nas bases de dados da Revista Tecnologística, características tradicionais e serviços comumente oferecidos pelos OLs no Brasil. Elas são as variáveis de controle do estudo, pois não constituem insumos ou produtos para os processos, mas, sim, atributos destes, num total de 25 variáveis contextuais. No Quadro 2, é apresentada a lista das variáveis de controle binárias, isto é, que apresentam escala dummy, utilizadas na pesquisa. Essas variáveis são terminais, de forma que assumem o valor de uma unidade caso a observação possua a característica mencionada e, valor nulo, caso contrário. É sabido que são necessárias $k-1$ variáveis dummy para representar uma variável com $k$ categorias (LEVINE et al., 2007). A categoria-base é a própria ausência da característica. Além dessas variáveis, deve ser mencionada a única exceção: o tempo de mercado, medido em meses (escala métrica).

Foi utilizado o software econométrico LIMDEP 9.0 para efetuar a regressão Tobit com dados em painel incompleto, considerando-se o modelo de efeitos aleatórios. Os resultados foram ajustados em função da heterocedasticidade gerada pelo fato de os grupos terem diferentes tamanhos (GREENE, 2007). Quanto ao nível de significância aceitável, estabeleceu-se a faixa entre 0,05 e 0,10 , adotada em pesquisas exploratórias em logística (MENTZER; FLINT, 1997; WANKE; HIJJAR, 2009). Na Tabela 3, são apresentados os resultados da regressão Tobit.

Quadro 2. Variáveis categóricas utilizadas na pesquisa*.

\begin{tabular}{|c|c|c|c|c|}
\hline Certificação ISO & $\begin{array}{l}\text { Consultas pela } \\
\text { internet }\end{array}$ & $\begin{array}{l}\text { Controle de } \\
\text { estoque }\end{array}$ & $\begin{array}{l}\text { Desenvolvimento } \\
\text { de projetos }\end{array}$ & Distribuição \\
\hline Embalagem & ERP & $\begin{array}{c}\text { Escritórios no } \\
\text { exterior }\end{array}$ & $\begin{array}{l}\text { Gerenciamento } \\
\text { intermodal }\end{array}$ & $\begin{array}{c}\text { Desembaraço } \\
\text { aduaneiro }\end{array}$ \\
\hline JIT & Logística reversa & Milk run & Montagem de kits & Porta a porta \\
\hline Raio de atuação local & $\begin{array}{l}\text { Raio de atuação } \\
\text { regional }\end{array}$ & $\begin{array}{l}\text { Rastreamento por } \\
\text { rádio próprio }\end{array}$ & $\begin{array}{l}\text { Rastreamento por } \\
\text { rádio terceirizado }\end{array}$ & $\begin{array}{l}\text { Rastreamento por } \\
\text { satélite terceirizado }\end{array}$ \\
\hline $\begin{array}{l}\text { Rastreamento por } \\
\text { satélite próprio }\end{array}$ & $\begin{array}{l}\text { Roteirizadores } \\
\text { próprios }\end{array}$ & Suporte fiscal & Transferência & WMS \\
\hline
\end{tabular}

*1 = apresenta a característica; 0 = não apresenta a característica.

Tabela 3. Resultados da regressão Tobit para dados em painel incompleto.

\begin{tabular}{|c|c|c|c|c|c|}
\hline \multirow{2}{*}{ Variável } & \multicolumn{5}{|c|}{ Regressão Tobit reestimada - Efeitos aleatórios ( $\left.{ }^{*}\right)$} \\
\hline & Coeficiente & Erro padrão & b/Er.Pd & $\mathrm{P}[\mathrm{Z}>\mathrm{z}]$ & Média de X \\
\hline Rastreamento por rádio terceirizado & .11803753 & .07160034 & 1.649 & .0992 & .68604651 \\
\hline Certificação ISO & .15098180 & .06303020 & 2.395 & .0166 & .57558140 \\
\hline ERP & .19309029 & .06835795 & 2.825 & .0047 & .74418605 \\
\hline Consultas pela internet & .14728202 & .07944126 & 1.854 & .0637 & .83720930 \\
\hline Tempo & .01604009 & .00501664 & 3.197 & .0014 & 9.02325581 \\
\hline Controle de estoque & .20318136 & .14223391 & 1.429 & .1531 & .93023256 \\
\hline Montagem de kits & -.20714991 & .11909732 & -1.739 & .0820 & .86627907 \\
\hline Sigma (v) & .26908893 & .02879359 & 9.345 & .0000 & \\
\hline Sigma $(u)$ & .24113454 & .04720093 & 5.109 & .0000 & \\
\hline \multirow{2}{*}{ Variável } & \multicolumn{5}{|c|}{ Efeitos Marginais (**) } \\
\hline & Coeficiente & Erro padrão & b/Er.Pd & $\mathrm{P}[\mathrm{Z}>\mathrm{z}]$ & Média de $X$ \\
\hline Rastreamento por rádio terceirizado & .10886679 & .06508581 & 1.673 & .0944 & .68604651 \\
\hline Certificação ISO & .13925151 & .07876336 & 1.768 & .0771 & .57558140 \\
\hline ERP & .17808843 & .09537775 & 1.867 & .0619 & .74418605 \\
\hline Consultas pela internet & .13583917 & .07082990 & 1.918 & .0551 & .83720930 \\
\hline Tempo & .01479388 & .07409954 & .200 & .8418 & 9.02325581 \\
\hline Controle de estoque & .18739550 & .07644378 & 2.451 & .0142 & .93023256 \\
\hline Montagem de kits & -.19105572 & .07124225 & -2.682 & .0073 & .86627907 \\
\hline Sigma (v) & .000000 & \multicolumn{4}{|c|}{ (Parâmetro fixo) } \\
\hline
\end{tabular}


Os resultados apresentados na Tabela 3 confirmam o impacto dos processos de coordenação na cadeia de suprimentos e, em particular, das Tls no aumento da eficiência de escala dos OLs brasileiros. Mais especificamente, merece destaque a adoção das seguintes tecnologias: rastreamento de rádio (terceirizado), ERP e consultas via internet sobre o status da carga em trânsito. A padronização dos processos de negócio pela certificação ISO também parece favorecer o aumento da eficiência de escala na indústria de Ols.

0 controle de estoques, por sua vez, apresentou efeito marginal significativo. Os resultados obtidos indicam que a sua presença provoca um aumento médio de 18,7 pontos percentuais na eficiência de escala dos OLs. Uma possivel justificativa para esse efeito é o fato de que o controle de estoques permite maior integração do fluxo de produtos do cliente com os recursos de transporte e de armazenagem dos OLs necessários à sua movimentação.

Deve ser ressaltado que o tempo de mercado dos OLs também tem impacto positivo na eficiência de escala. Além do acúmulo de experiências por operar por um período maior no mercado, deve-se levar em conta o fato de que o relacionamento entre a empresa contratante e o OL estreita-se, permitindo melhor coordenação dos recursos com as demandas dos clientes (BATHNAGAR; SOHAL; MILLEN, 1999).

Por último, a montagem de kits impacta negativamente na eficiência de escala dos OLs, possivelmente pela contratação de mão de obra adicional necessária para prestação desse tipo de serviço, vis-à-vis o retorno obtido.

\section{Conclusões}

Neste estudo, um modelo de DEA em dois estágios é utilizado não apenas para avaliar a eficiência de escala da indústria de OLs no Brasil, mas também para identificar seus principais determinantes. Os resultados corroboram as evidências na literatura de que mecanismos de coordenação na cadeia de suprimentos, como o uso de Tls e o controle de estoques, favorecem uma alocação mais racional dos recursos dos OLs (inputs) à demanda dos clientes (outputs) e, consequentemente, uma operação próxima do tamanho de escala mais produtivo, tendo como pano de fundo a disponibilização de informação em tempo real.

Os resultados apresentados trazem uma contribuição de caráter prático para o setor de OLs no Brasil. Mais precisamente, o estudo permite que gestores e investidores utilizem os resultados apresentados na Tabela 3 como apoio à tomada de decisão. Diversos direcionadores foram validados estatisticamente, revelando áreas onde há espaço não só para mais investimentos, mas também para desenvolvimento de futuras pesquisas que permitam compreender melhor a relação desses direcionadores com a eficiência de escala do setor.

Os OLs brasileiros se voltam cada vez mais para a ampliação da oferta de serviços, com constante redução de custos, na intenção de fazer frente a um quadro cada vez mais competitivo. Com isso, é de se esperar que os OLs recorram frequentemente a formas de avaliar seu desempenho nessa expansão, tanto quantitativa quanto qualitativamente. A estrutura utilizada no presente artigo pode ser aplicada como ferramenta em ambos os sentidos. Ela modela a situação do OL no momento da aplicação, auxiliando o direcionamento de recursos para áreas críticas que impactam significativamente no desempenho. 0 modelo previamente construído pode ser modificado de forma simples, acompanhando a dinâmica do quadro da empresa e apresentando resultados atualizados.

\section{Referências}

BANKER, R. D. Maximum likelihood, consistency and DEA: a statistical foundation. Management Science, v. 39, n. 10, p. 1265-1273, 1993

BANKER, R. D.; CHARNES, A.; COOPER, W. W. Some models for the estimation of technical and scale inefficiencies in data envelopment analysis. Management Science, v. 30, n. 9, p. 1078-1092, 1984.

BANKER, R. D.; NATARAJAN, R. Evaluating contextual variables affecting productivity using data envelopment analysis. Operations Research, v. 56, n. 1, p. 48-58, 2008.

BANKER, R. D.; NATARAJAN, R. Statistical tests based on DEA efficiency scores. In: COOPER, W. W.; SEIFORD, L. M.; $\mathrm{ZHU}, \mathrm{J}$. (Eds.). Handbook on data envelopment analysis. Boston: Kluwer International Series, 2004. p. 299-321.

BATHNAGAR, R.; SOHAL, A. S.; MILLEN, R. Third party logistics services: a Singapore perspective. International Journal of Physical Distribution \& Logistics Management, v. 29 , n. 9 , p. 569-587, 1999.

CHARNES, A.; COOPER, W. W.; RHODES, E. Measuring efficiency of decision-making units. European Journal of Operational Research, v. 2, n. 6, p. 429-444, 1978.

CHOU, S.; CHANG, Y. The implementation factors that influence the ERP (enterprise resource planning) benefits. Decision Support Systems, v. 46, n. 1, p. 149-157, 2008.

COOK, W. D.; SEIFORD, L. M. Data envelopment analysis (DEA): thirty years on. European Journal of Operational Research, v. 192, n. 1, p. 1-17, 2009

COOPER, W. W.; SEIFORD, L. M.; TONE, K. Data envelopment analysis: a comprehensive text with models, applications, references and DEA-solver software. New York: Springer, 2007.

DULÁ, J. H.; HELGASON, R. V. A new procedure for identifying the frame of the convex hull of a finite collection of 
points in multidimensional space. European Journal of Operational Research, v. 92, n. 2, p. 352-367, 1996.

FIGUEIREDO, K. F.; MORA, D. M. A segmentação dos operadores logísticos no mercado brasileiro de acordo com suas capacitações para oferecer serviços. RAC-Eletrônica, v. 3, n. 1, p. 123-141, 2009.

FLEURY, P. F. Vantagens competitivas e estratégicas do uso de operadores logísticos. In: FLEURY, P. F.; WANKE, P.; FIGUEIREDO, K. F. Logística Empresarial: a perspectiva Brasileira. São Paulo: Atlas, 2000. p. 133-142.

FLEURY, P. F.; RIBEIRO, A. F. M. A indústria de provedores de serviços logísticos no Brasil. In: FlGUEIREDO, K. F.; FLEURY, P. F.; WANKE, P. Logística e gerenciamento da cadeia de suprimentos: planejamento do fluxo e dos recursos. São Paulo: Atlas, 2003. p. 302-312.

FRIED, H. O. et al. Accounting for environmental effects and statistical noise in data envelopment analysis. Journal of Productivity Analysis, v. 17, n. 1/2, p. 157-174, 2002.

GOLANY, B.; ROLL, Y. An application procedure for DEA. Omega, v. 17, n. 3, p. 237-250, 1989.

GOMES, E. G. et al. Efficiency and sustainability assessment for a group of farmers in the Brazilian Amazon. Annals of Operations Research, v. 69, n. 1, p. 167-182, 2009.

GREEN, R.; DOYLE, J.; COOK, W. D. Efficiency bounds in data envelopment analysis. European Journal of Operational Research, v. 89, n. 3, p. 482-490, 1996.

GREENE, W. H. LIMDEP version 9.0: econometric modeling guide. New York: Econometric Software, 2007.

HAMDAN, A.; ROGERS, K. J. Evaluating the efficiency of 3PL logistics operations. International Journal of Production Economics, v. 113, n. 1, p. 235-244, 2007.

LEVINE, D. M. et al. Estatística: teoria e aplicações. São Paulo: LTC, 2007.

LEWIN, A.; MOREY, R.; COOK, T. Evaluating the administrative efficiency of courts. Omega, v. 10, n. 4, p. 401-411, 1982.

LIEB, R. C. The 3PL industry: where it's been, where it's going. Supply Chain Management Review, v. 9, n. 6, p. 20-27, 2005.

LIEB, R. C.; LIEB, K. J. Why 3PLs need to build their brand? Supply Chain Management Review, v. 12, n. 8, p. 46-52, 2008.

LIN, L. C.; TSENG, C. C. Operational performance evaluation of major container ports in the Asia-Pacific region. Maritime Policy \& Management, vol. 34, n. 6, p. 535-551, 2007.

LO, C. K. Y.; YEUNG, A. C. L.; CHENG, T. C. E. ISO 9000 and supply chain efficiency: empirical evidence on inventory and account receivable days. International Journal of Production Economics, v. 118, n. 2, p. 367-374, 2009.

MARINO, S. Mercado promissor. Revista Tecnologística, v. 19, n. 151, p. 68-73, 2008.

MASON, S. J. et al. Integrating the warehousing and transportation functions of the supply chain. Transportation Research Part E - Logistics \& Transportation Review, v. 39E, n. 2, p. 141-159, 2003.

MENTZER, J. T.; FLINT, D. J. Validity in logistics research. Journal of Business Logistics, v. 18, n. 1, p. 199-216, 1997.

MIN, H.; J0O, S. J. Benchmarking the operational efficiency of third party logistics providers using data envelopment analysis. Supply Chain Management: An International Journal, v. 11, n. 3, p. 259-265, 2006.

MIN, H.; PARK, B. l. Evaluating the inter-temporal efficiency trends of international container terminals using data envelopment analysis. International Journal of Integrated Supply Management, v. 1, n. 3, p. 258-277, 2005.

NG, B.; FERRIN, B. G.; PEARSON, J. N. The role of purchasing/ transportation in cycle time reduction. International Journal of Operations \& Production Management, v. 17, n. 6, p. 574-591, 1997.

ODECK, J.; ALKADI, A. Evaluating efficiency in the Norwegian bus industry using data envelopment analysis. Transportation, v. 28, n. 3, p. 211-232, 2001.

PACHECO, R. R.; FERNANDES, E. Managerial efficiency of Brazilian airports. Transportation Research Part A, v. 37, n. 8, p. 667-680, 2003.

PANAYIDES, P. M. et al. A critical analysis of DEA applications to seaport economic efficiency measurement. Transport Reviews, v. 29, n. 2, p. 183-206, 2009.

PARK, H. M. Linear regression models for panel data using SAS, Stata, LIMDEP, and SPSS. Disponível em: <www. indiana.edu/ statmath>. Acesso em: 15 abr. 2005.

ROSS, A. D.; DROGE, C. An analysis of operations efficiency in large-scale distribution systems. Journal of Operations Management, v. 21, n. 6, p. 673-688, 2004.

SCHEFCZYK, M. Operational performance of airlines: an extension of traditional measurement paradigms. Strategic Management Journal, v. 14, n. 4, p. 301-317, 1993.

SIMAR, L.; WILSON, P. W. Estimation and inference in two-stage, semiparametric models of production processes. Journal of Econometrics, v. 136, n. 1, p. 31-64, 2007.

SOUZA, G. S. et al. Economic efficiency of Embrapa's research centers and the influence of contextual variables. Pesquisa Operacional, v. 27, p. 15-26, 2007.

SOUZA, G. S.; STAUB, R. B. Two-stage inference using data envelopment analysis efficiency measurements in univariate production models. International Transactions in Operational Research, v. 14, n. 3, p. 245-258, 2007.

STEFANSSON, G.; LUMSDEN, K. Performance issues of smart transportation management systems. International Journal of Productivity and Performance Management, v. 58, n. 1, p. 55-70, 2009.

TURNER, H.; WINDLE, R.; DRESSNER, M. North American containerport productivity: 1984-1997. Transportation Research Part E, v. 40, n. 4, p. 339-356, 2004.

UNIVERSIDADE FEDERAL DO RIO DE JANEIRO. Centro de Estudos em Logística - CEL. Panorama logístico CEL/ COPPEAD: terceirização logística no Brasil. Rio de Janeiro: UFRJ, 2009.

WAGNER, J. M.; SHIMSHAK, D. G. Stepwise selection of variables in data envelopment analysis: procedures and managerial perspectives. European Journal of Operational Research, v. 180, n. 1, p. 57-67, 2007.

WANG, T. F.; SONG, D. W.; CULLINANE, K. The applicability of data envelopment analysis to efficiency measurement of container ports. Cidade do Panamá: IAME, 2002.

WANKE, P. F. Logística, gerenciamento de cadeias de suprimento e estratégia logística. In: FIGUEIREDO, K. F.; FLEURY, P. F.; WANKE, P. Logística e gerenciamento 
da cadeia de suprimentos. planejamento do fluxo e dos recursos. São Paulo: Atlas, 2003. p. 27-47.

WANKE, P. F.; ARKADER, R.; HIJJAR, M. F. Logistics sophistication, manufacturing segments and the choice of logistics providers. International Journal of Operations \& Production Management, v. 27, n. 5, p. 542-559, 2007.

WANKE, P. F.; HIJJAR, M. F. Exportadores brasileiros: estudo exploratório das percepções sobre a qualidade da infraestrutura logística. Produção, v. 19, n. 1, p. 143-162, 2009.

WILDING, R.; JURIADO, R. Customer perceptions on logistics outsourcing in the European consumer goods industry. International Journal of Physical Distribution \& Logistics Management, v. 34, n. 8, p. 28-48, 2004.
YOUNGBERG, E.; OLSEN, D.; HAUSER, K. Determinants of professionally autonomous end user acceptance in an enterprise resource planning system environment. International Journal of Information Management, v. 29, n. 2, p. 138-144, 2009.

ZHOU, G. et al. Evaluating the comparative efficiency of Chinese third-party logistics providers using data envelopment analysis. International Journal of Physical Distribution \& Logistics Management, v. 38, n. 4, p. 262-279, 2008.

ZHU, J. Quantitative models for performance evaluation and benchmarking: data envelopment analysis with spreadsheets and DEA excel solver. New York: Springer, 2003.

\title{
Determinants of scale efficiency in the Brazilian 3PL industry
}

\begin{abstract}
This paper focuses on the third-party logistics (3PL) industry in Brazil which, facing a highly competitive scenario, offers its clients a broad range of services and information technologies in pursuit of higher levels of efficiency. Its main objective is to determine the variables that significantly impact on the 3PL scale efficiency by means of a two-stage Data Envelopment Analysis (DEA). Data (2001-2008) from the annual survey developed by Revista Tecnologistica were used in an unbalanced panel data model. The inputs and outputs required for this analysis were identified as well as the contextual variables that may impact on the 3PL scale efficiency. The results corroborate the evidence in the literature on the role of coordination processes in the logistics performance.
\end{abstract}

\section{Keywords}

DEA. 3PL. Scale efficiency. Data panel. 